30.P1

\title{
Functional Properties of Subretinal Transplants in Rabbit
}

\author{
A.R. Adolph ${ }^{1}$, C.L. Zucker ${ }^{1}$, B. Ehinger ${ }^{2}$ and A. Bergström ${ }^{2}$ \\ ${ }^{1}$ Schepens Eye Research Institute and Harvard Medical School, Boston 02114, MA, USA; \\ ${ }^{2}$ Department of Ophthalmology, University of Lund, Sweden
}

There has been a rapid growth in research focused on retinal transplantation as a strategy for restoration of or rescue of function in retinas rendered afunctional by disease or trauma. This strategy has been partially stimulated by recent transplantation efforts in brain related to neurodegenerative diseases such as Parkinson's, Huntington's, and Alzheimer's. These latter efforts have been hindered by a dearth of information regarding the specific properties of the inputs, intrinsic processing and outputs within the brain underlying these diseases. This is especially significant when attempting to study correlated functional and structural properties of the implanted tissue. In contrast to such studies, this research utilizes the retina, of which there is greater knowledge about the inputs (complex light patterns), intra-retinal processing and anatomical micro-circuitry, and the output (patterns of ganglion cell activity). Our research demonstrates, for the first time, organized, complex physiological function and its underlying neuronal microcircuitry in mammalian subretinal transplants.
Grafts which develop from transplanting fragments of embryonic donor retina into the subretinal space of a mature host exhibit atypical retinal organization. A combination of light and electron microscopy plus intracellular dye marking has shown that the grafts often develop regions containing "rosettes" of photoreceptors surrounded by appropriately layered retinal elements. The transplants contain abundantly the specific cellular and synaptic components which are necessary for phototransduction and for the processing of visual information in a fashion similar to that carried out by normal retinas.

In testing the effect of this organization on graft functional properties, we isolated the graft to avoid contamination of graft responses by host retinal activity. Light-driven responses were recorded by microelectrodes from this preparation maintained in vitro. Local electroretinograms and spike-like responses to flashed spot stimuli is transient ON or ON-OFF. The amplitudes and durations of these response components depend on stimulus diameter, sometimes

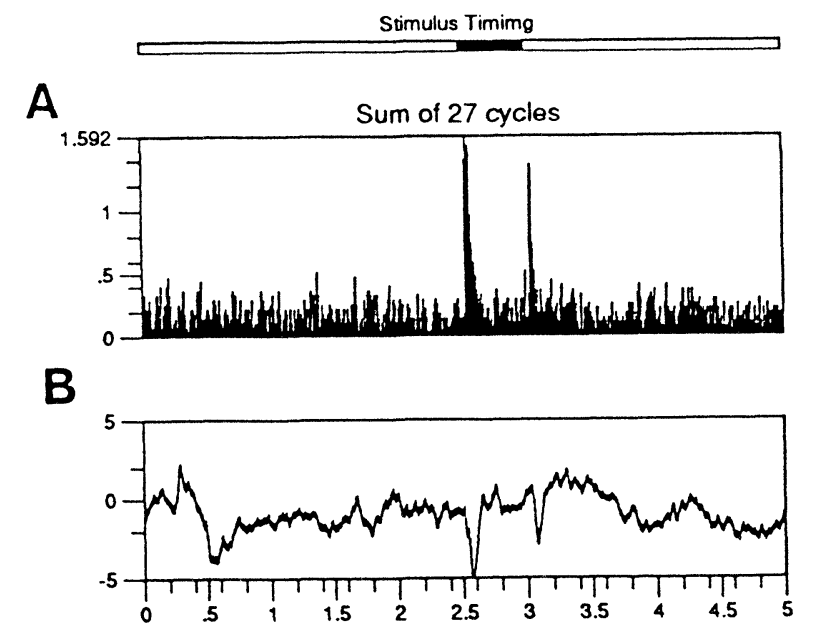

Fig. 1 
in a way suggesting center-surround receptive field organization and spatial tuning. Response amplitudes are also a function of light intensity. The local electroretinogram transient hyperpolarizations at light-ON and -OFF may reflect light-induced amacrine cell activity (M-waves). Thus, these rabbit retinal grafts contain the neural components, local synaptic circuitry and exhibit the electrophysiological responses necessary to process complex visual information.

\section{CONCLUSIONS}

Based on intracellular filling, light and electron microscopy, retinal grafts contain the major neuronal cell types present in normal retinas, with the possible exception of ganglion cells. These neuronal elements are organized at the cellular and synaptic levels in a fashion appropriate for retinal function. Retinal grafts show light responsiveness representing photoreceptor function and localized visual information processing. The spatial properties of the responses indicate possible center-surround functional organization in the outer plexiform layer. The temporal properties, such as ON-OFF light responses, suggest inner plexiform layer signal processing. Taken together, these results demonstrate a degree of functional ability and capacity in retinal transplants required for useful therapeutic purposes. 

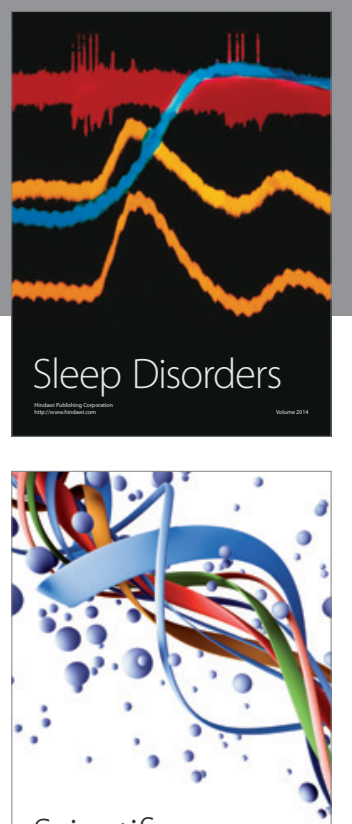

Scientifica
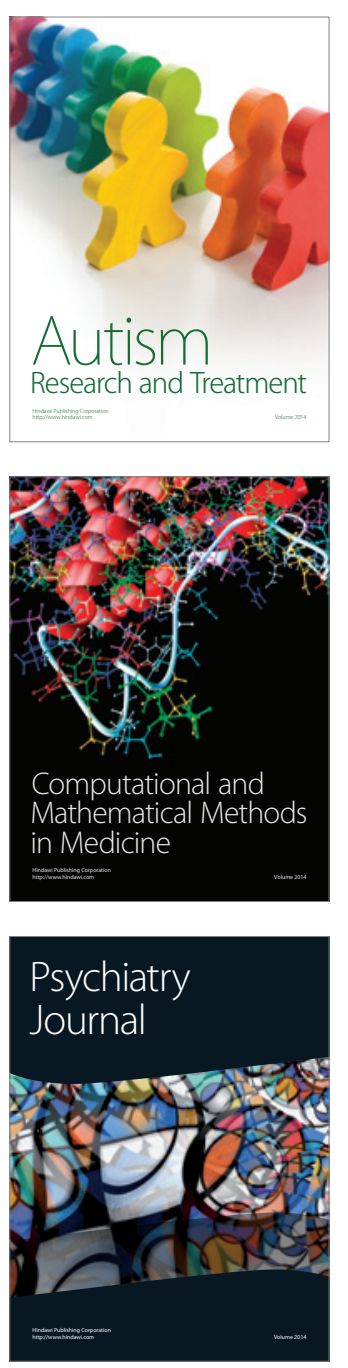
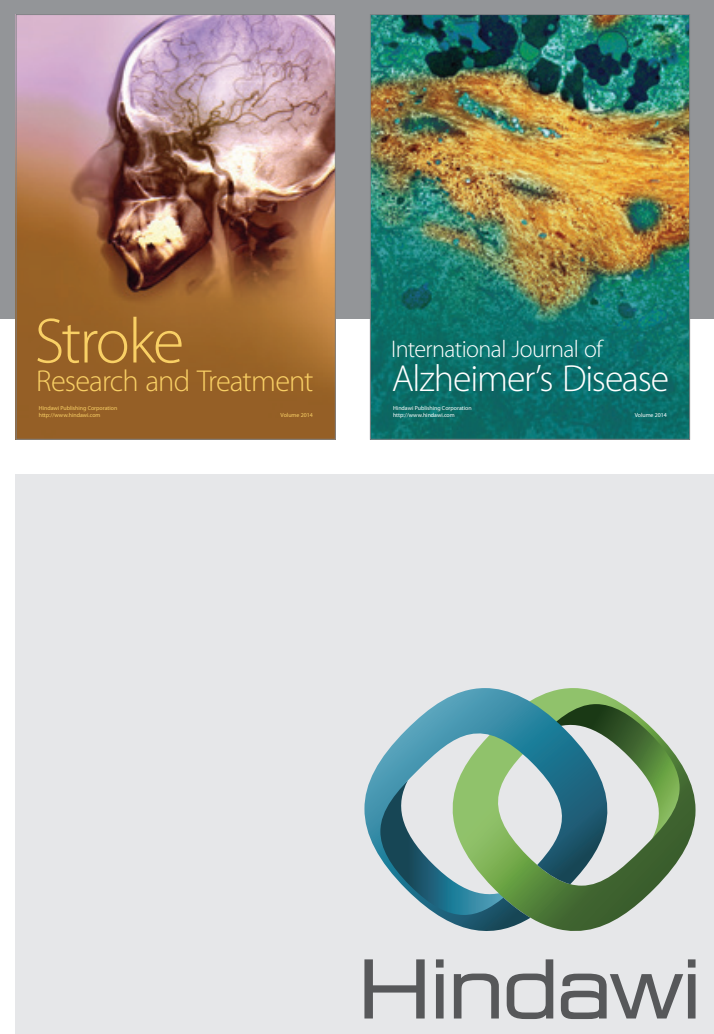

Submit your manuscripts at

http://www.hindawi.com
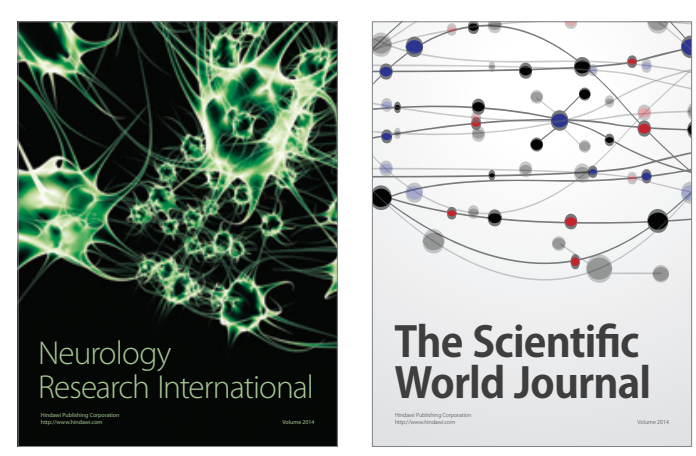

The Scientific World Journal

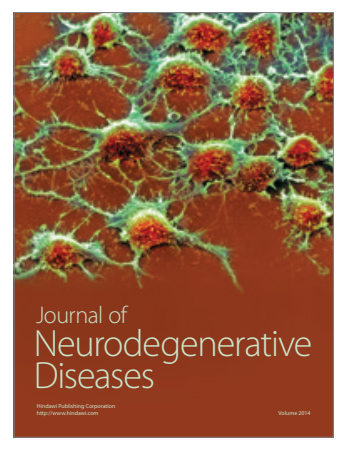

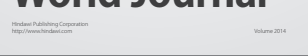

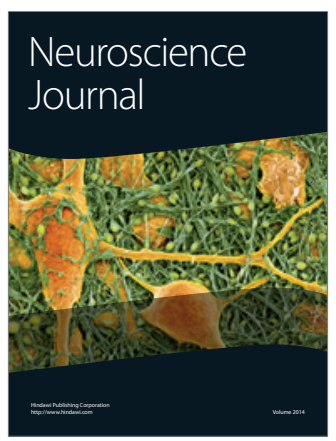

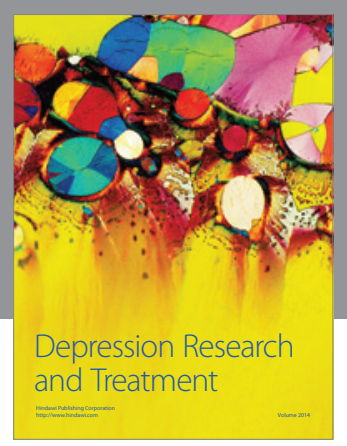
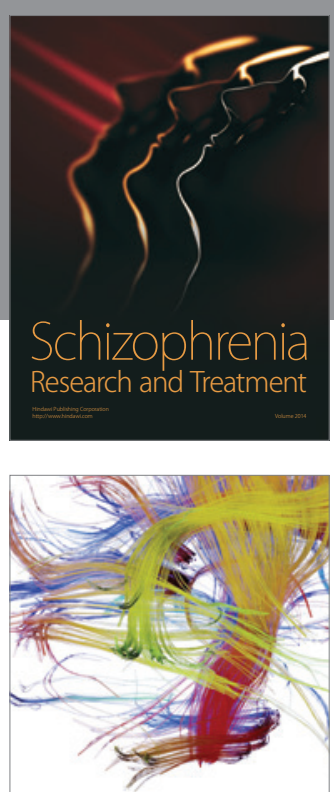

Brain Science

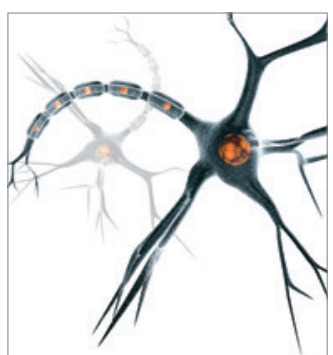

Neural Plasticity
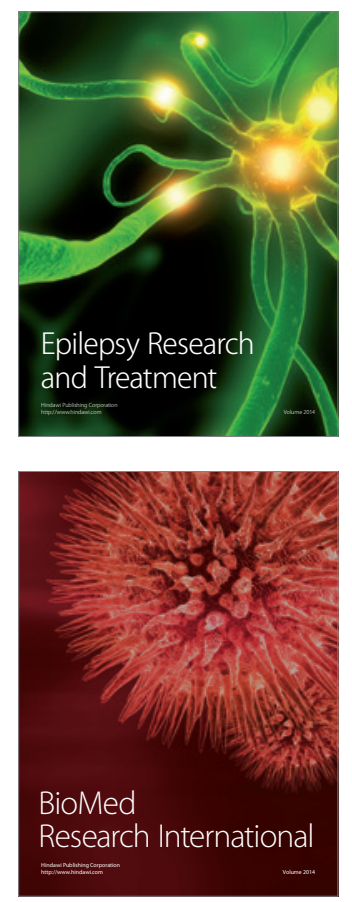

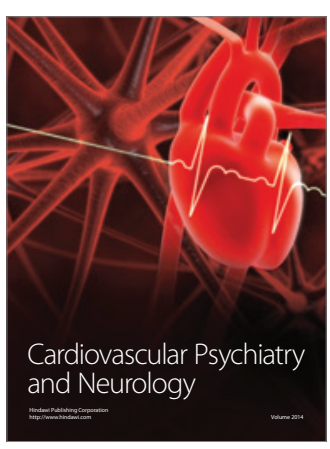

Parkinson's

Disease
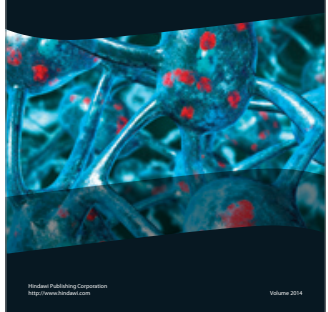